\title{
Refraction traveltime tomography using damped monochromatic wavefield
}

\author{
Sukjoon Pyun ${ }^{1}$, Changsoo Shin ${ }^{1}$, Dong-Joo $\mathrm{Min}^{2}$, and Taeyoung $\mathrm{Ha}^{3}$
}

\begin{abstract}
For complicated earth models, wave-equation-based refraction-traveltime tomography is more accurate than ray-based tomography but requires more computational effort. Most of the computational effort in traveltime tomography comes from computing traveltimes and their Fréchet derivatives, which for ray-based methods can be computed directly. However, in most wave-equation traveltime-tomography algorithms, the steepest descent direction of the objective function is computed by the backprojection algorithm, without computing a Fréchet derivative directly.

We propose a new wave-based refraction-traveltimetomography procedure that computes Fréchet derivatives directly and efficiently. Our method involves solving a damped-wave equation using a frequency-domain, finite-element modeling algorithm at a single frequency and invoking the reciprocity theorem. A damping factor, which is commonly used to suppress wraparound effects in frequency-domain modeling, plays the role of suppressing multievent wavefields. By limiting the wavefield to a single first arrival, we are able to extract the first-arrival traveltime from the phase term without applying a time window. Computing the partial derivative of the damped wave-equation solution using the reciprocity theorem enables us to compute the Fréchet derivative of amplitude, as well as that of traveltime, with respect to subsurface parameters. Using the Marmousi-2 model, we demonstrate numerically that refraction traveltime tomography with large-offset data can be used to provide the smooth initial velocity model necessary for prestack depth migration.
\end{abstract}

\section{INTRODUCTION}

Refraction surveys were first used by seismologists in the early twentieth century to investigate the deep structure of the earth. Following that, exploration geophysicists succeeded in delineating shallow salt bodies for oil exploration. With the enhancement of acquisition techniques and improved equipment, reflection surveys have replaced refraction surveys in hydrocarbon exploration. Furthermore, since the depth penetration of refracted waves is limited by the source-receiver offset in a reflection seismic survey, refracted waves have been employed mainly to investigate the shallow subsurface for static corrections. Refracted waves are interpreted chiefly by traveltime tomographic inversion.

In the early period, refraction-traveltime tomography generally relied on the ray-tracing method to compute first-arrival traveltimes and their Fréchet derivatives. In ray-based refraction tomography, the Fréchet derivative of traveltime with respect to slowness is defined as the distance traversed by the ray. This simplicity makes geophysicists favor ray-based refraction-traveltime tomography, and consequently a variety of ray-tracing refraction tomography methods have been developed (Hampson and Russell, 1984; Schneider and Kuo, 1985; White, 1989; Zhu and McMechan, 1989; Docherty, 1992; Qin et al., 1993; Cai and Qin, 1994; Stefani, 1995; Shtivelman, 1996; Zhang and Toksöz, 1998; Shin et al., 1999). However, ray-tracing traveltime tomography, which is a high-frequency approximation, sometimes fails to compute Fréchet derivatives due to shadow zones.

In order to overcome this weakness of the ray-based traveltime tomography, wavepath methods (Woodward and Rocca, 1988; Woodward, 1992; Luo and Schuster, 1990, 1991; Schuster and Quintus-Bosz, 1993) and Fresnel volume methods (Vasco et al., 1995) have been used in traveltime tomographic inversion. Luo and Schuster (1991) devised a

Manuscript received by the Editor April 16, 2003; revised manuscript received August 19, 2004; published online March 22, 2005.

${ }^{1}$ Seoul National University, Division of Civil, Urban and Geosystem Engineering, San 56-1, Shillim-Dong, Gwanak-Gu, Seoul, 151-742, Korea. E-mail: pyunsj@gpl.snu.ac.kr; css@model.snu.ac.kr.

${ }^{2}$ Korea Ocean Research and Development Institute, 1270, Sa2-Dong, Sangrok-Gu, Ansan, Gyunngi, 426-744, Korea. E-mail: djmin@kordi.re.kr. ${ }^{3}$ Seoul National University, Department of Mathematical Science, San 56-1, Shillim-Dong, Gwanak-Gu, Seoul, 151-742, Korea. E-mail: tyha@math.snu.ac.kr.

(C) 2005 Society of Exploration Geophysicists. All rights reserved. 
wave-equation traveltime-inversion technique by introducing a connective function that relates the traveltime residual to the pressure seismogram. In their method, the steepest descent direction of the $L_{2}$ norm of traveltime residuals is computed by backprojecting seismogram residuals.

These approaches to wave-equation traveltime tomography, using a band-limited approximation, correctly describe high velocity-contrast models but incur high computational costs to compute wavepaths. Schuster and QuintusBosz (1993) enhance computational efficiency by computing wavepaths using the eikonal equation. Fresnel-volume traveltime tomography, which is a modified ray-tracing method, gives results similar to those of wave-equation tomography while incurring computational costs comparable to those of ray-tracing tomography. However, Fresnel-volume tomography is a high-frequency approximation and, therefore, sometimes fails near the source and receiver at low frequencies (Vasco et al., 1995).

Based on the merits of wave-equation traveltime tomography, we suggest a new refraction-traveltime tomographic inversion technique that efficiently computes the Fréchet derivatives of traveltime and amplitude using a monochromatic damped wavefield (e.g., Shin et al., 2003a). By introducing a damping factor inherent in frequency-domain modeling, we can choose the first-arrival traveltime without time windowing or tapering and explicitly calculate the Fréchet derivatives. Our method is similar to that of Woodward (1992) and Schuster and Quintus-Bosz (1993) in that we extract traveltimes from phase terms. The main difference between conventional wave-equation methods and our method is in the computation of Fréchet derivatives of traveltimes and amplitudes. In conventional wave-equation traveltime tomography, the steepest descent direction is computed by crosscorrelating forward-modeled wavefields and backprojected traveltime residuals. In our technique, Fréchet derivatives are computed by calculating the partial derivative of the damped monochromatic wavefields with respect to velocity. In our approach, we reduce computational costs required to compute Fréchet derivatives by applying the source-receiver reciprocity theorem (Shin et al., 2001b). We demonstrate our refractiontraveltime tomography algorithm by applying the velocitydepth model generated with our refraction-traveltime tomography to Kirchhoff prestack depth migration for the Marmousi-2 model (Martin et al., 2002) and reconfirm that the Kirchhoff prestack depth migration is sensitive to the initial model.

\section{CALCULATION OF TRAVELTIME AND ITS FRÉCHET DERIVATIVE}

In wave-equation-based traveltime tomography, it is important to efficiently compute traveltime and its Fréchet derivative. For an efficient traveltime-tomography method, we suggest using a damped monochromatic wave equation as Shin et al. (2003a) used to compute first-arrival traveltime. In this section, we briefly review the traveltime computation method of Shin et al. (2003a) and then explain how to explicitly compute Fréchet derivatives from the damped monochromatic wavefield.

When solving the wave equation by using the time-domain finite-element method, we need to solve the discretized matrix equation given by

$$
\mathbf{M u ̈}+\mathbf{K u}=\mathbf{f},
$$

where $\mathbf{M}$ is the mass matrix, $\mathbf{K}$ is the stiffness matrix, $\mathbf{u}$ is the pressure or displacement vector, $\ddot{\mathbf{u}}$ is the second-order partial derivative of the pressure or displacement vector with respect to time, and $\mathbf{f}$ is the source vector (Marfurt, 1984).

In the temporal Fourier domain, we can rewrite equation 1 as

$$
\mathbf{S} \tilde{\mathbf{u}}=\tilde{\mathbf{f}}
$$

with

$$
\mathbf{S}=\mathbf{K}-\omega^{2} \mathbf{M},
$$

where $\mathbf{S}$ is the complex impedance matrix and $\tilde{\mathbf{u}}(\omega)$ and $\tilde{\mathbf{f}}(\omega)$ are the Fourier transforms of $\mathbf{u}(t)$ and $\mathbf{f}(t)$, respectively, expressed as

$$
\tilde{\mathbf{u}}(\omega)=\int_{-\infty}^{\infty} \mathbf{u}(t) e^{-i \omega t} d t
$$

and

$$
\tilde{\mathbf{f}}(\omega)=\int_{-\infty}^{\infty} \mathbf{f}(t) e^{-i \omega t} d t .
$$

In order to obtain the frequency-domain wave solution $\tilde{\mathbf{u}}$, we factor the impedance matrix $\mathbf{S}$ into upper and lower triangular matrices. We then obtain the frequency-domain wave solution $\tilde{\mathbf{u}}$ by forward and backward substitutions. In the frequency-domain modeling, we often use the complex angular frequency $\omega$ to suppress the wraparound effects:

$$
\omega=\omega^{*}+i \alpha,
$$

where $\omega^{*}$ is the real angular frequency and $\alpha$ is the wraparound suppression factor. The resulting wavefield in the time domain is equivalent to the wavefield damped by $e^{-\alpha t}$. By choosing the optimum wraparound suppression factor (Shin et al., 2003a), we were able to suppress all the energy following the first arrival and obtain a damped spike.

The damped spike-shaped wavefield can be expressed (Shin et al., 2003a) in the frequency domain as

$$
\tilde{u}\left(x, y, z, \omega^{*}\right)=A(x, y, z) e^{-\alpha \tau(x, y, z)} e^{i \omega^{*} \tau(x, y, z)},
$$

where $\tilde{u}\left(x, y, z, \omega^{*}\right)$ is the Fourier transformed wavefield, $\tau(x, y, z)$ is the traveltime from the source to a depth point in the subsurface, and $A(x, y, z)$ is the amplitude at the depth point in the subsurface.

By taking the logarithm of both sides of equation 7, we obtain

$\ln \tilde{u}\left(x, y, z, \omega^{*}\right)=\ln \left|A(x, y, z) e^{-\alpha \tau(x, y, z)}\right|+i \omega^{*} \tau(x, y, z)$.

Dividing the imaginary component of equation 8 by the real angular frequency $\omega^{*}$ gives us the traveltime $\tau(x, y, z)$ at each depth point. Although first-arrival traveltimes can be computed using an arbitrarily chosen angular frequency, Shin et al. (2003a) found that choosing a low frequency for traveltime computation avoids cycle-skipping effects.

For our refraction-traveltime tomography, we calculate the Fréchet derivatives using the damped-wave solutions in the 
frequency domain. In order to compute the Fréchet derivatives efficiently, we exploit source-receiver reciprocity as described by Shin et al. (2001b). To proceed, we express equation 7 as

$$
\begin{array}{r}
\tilde{u}_{i, j}(\omega)=A_{i, j} e^{-\alpha \tau_{i, j}} e^{i \omega \tau_{i, j}} \\
i=1,2, \ldots, n_{r} ; \quad j=1,2, \ldots, n_{s},
\end{array}
$$

where $i$ indicates the receiver number, $j$ denotes the shot number, $n_{r}$ is the number of receivers, and $n_{s}$ is the number of shots. Following Shin et al.'s (2001b) notation, we parameterize our subsurface by $N=N_{x} \times N_{z}$ elements, where $N_{x}$ and $N_{z}$ are the numbers of elements in the $x$ - and $z$-directions, respectively. We assume that the unknown model parameter vector $\mathbf{p}$ is defined as the velocity within each element, represented by $\mathbf{p}=\left[p_{1}, p_{2}, \ldots, p_{\ell}, \ldots, p_{N}\right]$. Since the wavefield can be expressed as a function of the velocity parameter (e.g., Shin, 1988), we differentiate $\tilde{u}_{i, j}$ with respect to the subsurface velocity parameter $p_{\ell}$. As a result, we obtain partial-derivative wavefields having the form

$$
\begin{aligned}
& \frac{\partial \tilde{u}_{i, j}}{\partial p_{\ell}}= \frac{\partial A_{i, j}}{\partial p_{\ell}} e^{-\alpha \tau_{i, j}} e^{i \omega \tau_{i, j}}+A_{i, j} e^{-\alpha \tau_{i, j}}(-\alpha) \frac{\partial \tau_{i, j}}{\partial p_{\ell}} e^{i \omega \tau_{i, j}} \\
&+A_{i, j} e^{-\alpha \tau_{i, j}} e^{i \omega \tau_{i, j}} i \omega \frac{\partial \tau_{i, j}}{\partial p_{\ell}} \\
& i=1,2, \ldots, n_{r} ; \quad j=1,2, \ldots, n_{s} ; \quad l=1,2, \ldots, N .
\end{aligned}
$$

In the process of computing the partial derivative wavefields with respect to each model parameter, we invoke the sourcereceiver reciprocity theorem (Shin et al., 2001b).

The next stage is to use these partial-derivative wavefields with respect to model parameters to calculate the Fréchet derivative of traveltime. Dividing both sides of equation 10 by equation 9 and rearranging gives

$$
\begin{gathered}
\frac{1}{\tilde{u}_{i, j}} \frac{\partial \tilde{u}_{i, j}}{\partial p_{\ell}}=\frac{1}{A_{i, j}} \frac{\partial A_{i, j}}{\partial p_{\ell}}-\alpha \frac{\partial \tau_{i, j}}{\partial p_{\ell}}+i \omega \frac{\partial \tau_{i, j}}{\partial p_{\ell}} \\
i=1,2, \ldots, n_{r} ; \quad j=1,2, \ldots, n_{s} ; \quad l=1,2, \ldots, N .
\end{gathered}
$$

We can extract the Fréchet derivative of traveltime from the imaginary part of equation 11.

As a byproduct of calculating the Fréchet derivative of traveltime, we can also compute the Fréchet derivative of the absolute amplitude from equation 11.

\section{VERIFICATION OF FRÉCHET DERIVATIVE}

To verify our algorithm for computing the derivative of traveltime, we compare the analytically calculated Fréchet derivative with the numerically computed Fréchet derivative (Lines and Treitel, 1984). By the finite-difference formula, the variation of traveltime $\tau_{i}$ with respect to the parameter $p_{j}$ is expressed by

$$
J_{i, j}=\frac{\partial \tau_{i}}{\partial p_{j}} \cong \frac{\tau_{i}\left(p_{j}+\Delta p_{j}\right)-\tau_{i}\left(p_{j}-\Delta p_{j}\right)}{2 \Delta p_{j}} .
$$

To verify the Fréchet derivative, we choose a simple homogeneous model (Figure 1) whose velocity is $2 \mathrm{~km} / \mathrm{s}$. The size of the model is $5 \mathrm{~km}$ wide by $0.5 \mathrm{~km}$ deep. We subdivide the model into 25000 elements, each of $10 \mathrm{~m}$ by $10 \mathrm{~m}$ size. We then perturb the element at $(x=2.5 \mathrm{~km}, z=0.1 \mathrm{~km})$ (Figure 1 ) and measure traveltimes along the surface.

Figures $2 \mathrm{a}, 2 \mathrm{~b}$, and $2 \mathrm{c}$ show analytic and finite-difference Fréchet derivatives where the source is located at $1.5 \mathrm{~km}$, $2.5 \mathrm{~km}$, and $4.5 \mathrm{~km}$, respectively. Note that the numerical Fréchet derivatives agree well with the analytic Fréchet derivatives. Note that in equation 11, the Fréchet derivative is generated by computing partial derivative wavefields that

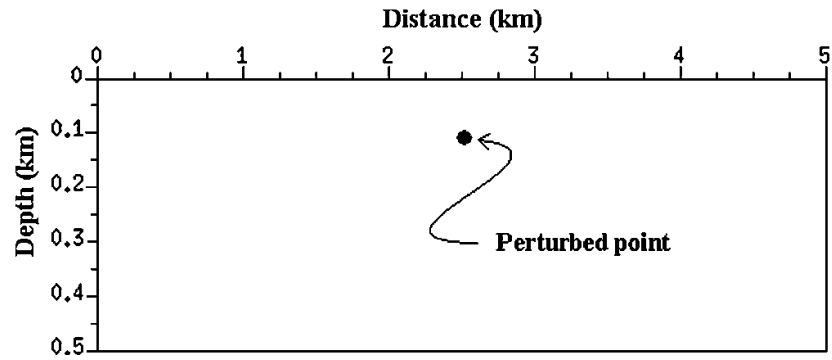

Figure 1. Velocity model for comparing the analytic Fréchet derivative with the numerical Fréchet derivative.
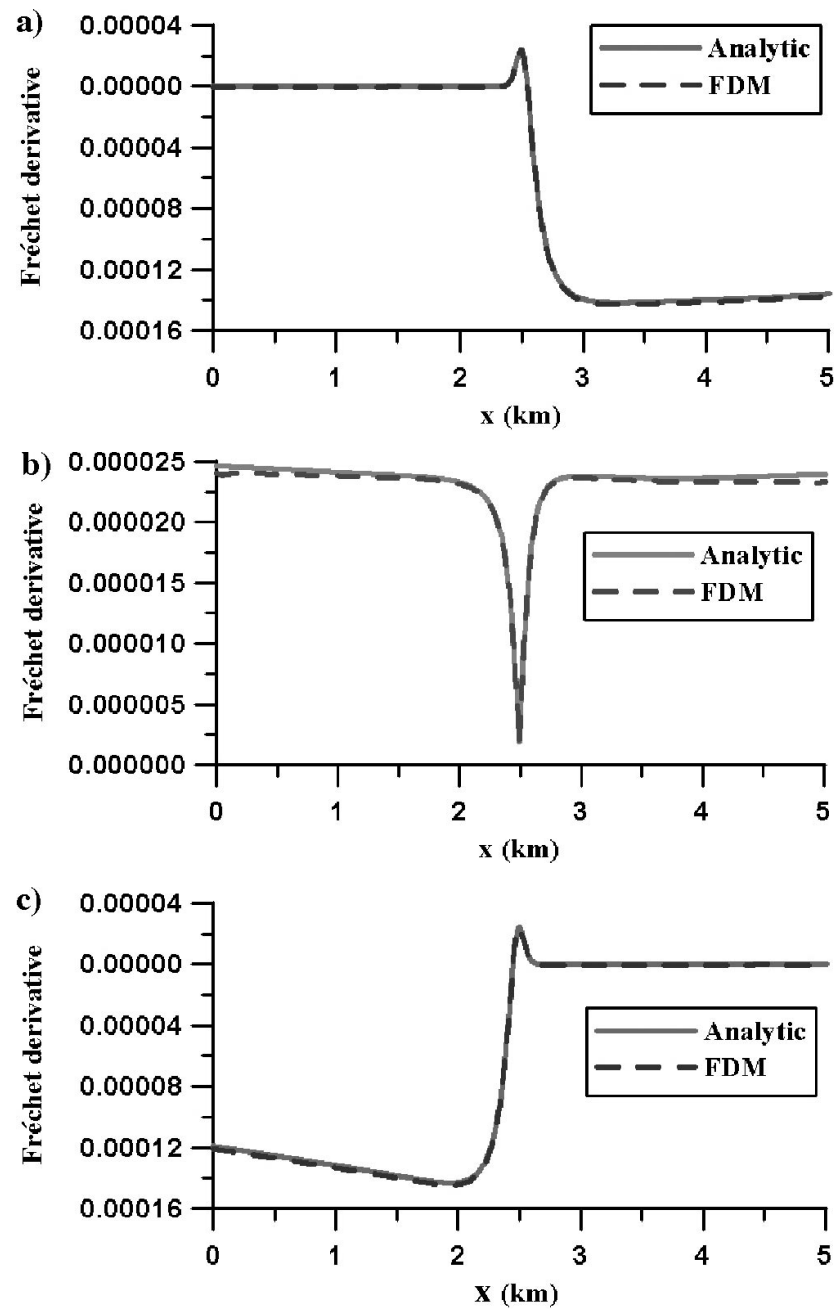

Figure 2. Comparison of the analytic and numerical Fréchet derivative of traveltime, where the shot is located at (a) 1.5 $\mathrm{km}$, (b) $2.5 \mathrm{~km}$, and (c) $4.5 \mathrm{~km}$, respectively. FDM = finitedifference method. 
are obtained by introducing virtual sources (e.g., Pratt et al., 1998; Shin and Chung, 1999). When a wavefield propagates from the shot point to a perturbed point, it acts as a virtual source and reradiates energy to all receivers at the surface. In this manner, we can compute Fréchet derivatives even for a homogeneous medium, thereby allowing us to start with a homogeneous model as an initial model in refraction-traveltime tomography.

We also check the Fréchet derivative of amplitude. In Figure 3, we compare analytically computed Fréchet derivatives of amplitude with numerically computed values for the homogeneous model shown in Figure 1. As shown in Figure 3, the numerically computed Fréchet derivatives of the amplitude are also compatible with the analytically computed ones.

\section{INVERSION ALGORITHM}

In traveltime-tomographic inversion using the least-squares method, we define the objective function as the $L_{2}$ norm of residual errors between the first-arrival traveltimes of the real
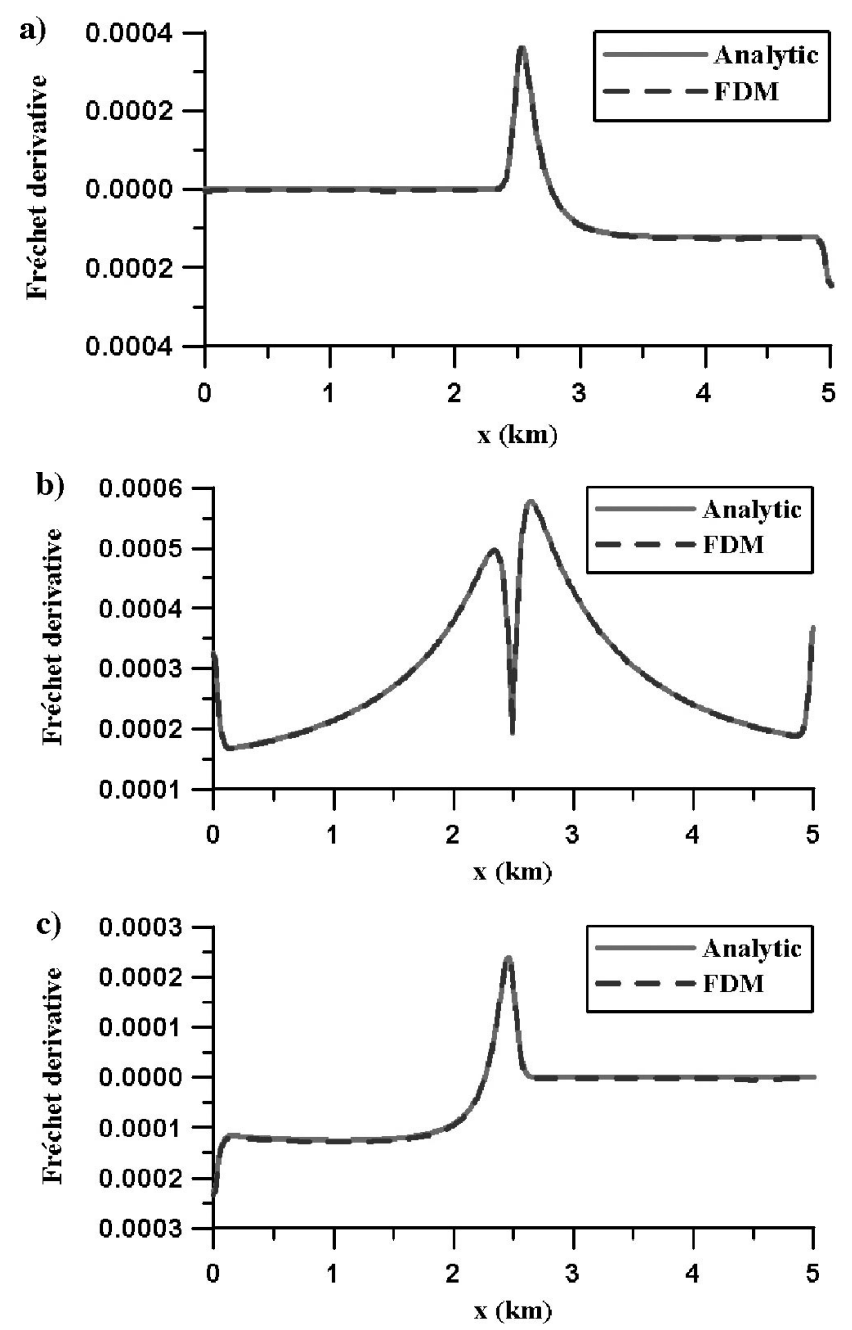

Figure 3. Comparison of the analytic and numerical Fréchet derivative of amplitude where the shot is located at (a) $1.5 \mathrm{~km}$, (b) $2.5 \mathrm{~km}$, and (c) $4.5 \mathrm{~km}$, respectively. FDM = finitedifference method. data and those obtained by numerical modeling:

$$
E(\mathbf{p})=\frac{1}{2} \Delta \boldsymbol{\tau}^{\mathbf{t}} \Delta \boldsymbol{\tau}
$$

and

$$
\Delta \tau=\tau_{\mathbf{d}}-\tau_{\mathbf{u}}
$$

where $\mathbf{p}$ is the material property vector, $\boldsymbol{\tau}_{\mathbf{d}}$ is the observed traveltime, $\tau_{\mathbf{u}}$ is the numerically computed traveltime, and the superscript $\mathbf{t}$ denotes the transpose.

In general, the least-squares method needs either direct or indirect estimates of the Fréchet-derivative. In this study, we choose the steepest descent method and update the velocity model by following the general iterative rule

$$
\mathbf{p}^{(k+1)}=\mathbf{p}^{(k)}-\alpha^{(k)} \nabla_{p} E^{(k)},
$$

where

$$
\nabla_{p} E^{(k)}=\frac{\partial E^{(k)}}{\partial \mathbf{p}}=\mathbf{J}^{\mathbf{t}} \Delta \boldsymbol{\tau}
$$

and $k$ is the iteration number, $\nabla_{p} E^{(k)}$ is the steepest descent direction normal to the objective function at the $k$ th iteration, $\alpha$ is the step length, and $\mathbf{J}^{\mathbf{t}}$ is the transpose of the Fréchetderivative matrix.

When using the Gauss-Newton or full Newton method, we can obtain the step length by inverting the full Hessian or an approximate Hessian and multiplying the steepest descent direction by the inverse of the Hessian. However, if the number of unknowns is large, the computation of the Hessian is a formidable task, even employing a PC cluster with MPI interprocess communication. Furthermore, calculating an inverse of the huge Hessian is also prohibitively expensive and sometimes suffers from the illposedness of the Hessian (Shin et al., 2001a). In our tomographic inversion, we efficiently regularize the steepest descent direction using the diagonals of the Hessian rather than the full Hessian or the approximate Hessian, since the Hessian is diagonally dominant. In order to avoid the illposedness of the Hessian, we also add the damping factor to the diagonals of the Hessian.

\section{EXAMPLE OF AN ISOLATED BLOCK EMBEDDED IN THE TWO-LAYERED MODEL}

In order to test our algorithm, we take an isolated block embedded in a two-layer model. Figure 4a shows the true model. The velocities of the first layer, second layer, and the block in the middle are $1.5 \mathrm{~km} / \mathrm{s}, 4.5 \mathrm{~km} / \mathrm{s}$ and $3 \mathrm{~km} / \mathrm{s}$, respectively. The size of the model is $3 \mathrm{~km}$ in horizontal distance and $0.5 \mathrm{~km}$ in depth. For the initial model, we assume a velocity model linearly increasing with the depth, ranging from $1.5 \mathrm{~km} / \mathrm{s}$ to $3.5 \mathrm{~km} / \mathrm{s}$, as shown in Figure $4 \mathrm{~b}$. We subdivide the velocity model into $300 \times 50$ elements. At the surface with intervals of $10 \mathrm{~m}, 301$ receivers are placed. Figure $4 \mathrm{c}$ displays the inverted model at the 600th iteration. Since we use a small step length, the convergence rate is very slow. Note that the inverted velocity model converges to the true model in Figure 4c. Figure 5a describes traveltimes computed for the true, the initial, and the inverted model; Figure $5 \mathrm{~b}$ shows the rms error of the traveltimes, which decreases from $61 \mathrm{~ms}$ at the initial stage to $1 \mathrm{~ms}$ at 600 iterations. 


\section{MARMOUSI-2 MODEL}

We also invert the traveltimes generated for the Marmousi2 model (Martin et al., 2002). Figure 6a shows the P-wave velocity model for the Marmousi-2 model. For simplicity, we peeled off the model's water layer. The horizontal distance of the model is $17 \mathrm{~km}$ and the depth is $3 \mathrm{~km}$. The lowest velocity is $1.028 \mathrm{~km} / \mathrm{s}$ in the gas-charged sand channel and gas-sand trap. The maximum velocity is $4.7 \mathrm{~km} / \mathrm{s}$ in the salt layer. The initial model for inverting the Marmousi- 2 model is shown in Figure $6 \mathrm{~b}$. At the surface, the velocity is $1.5 \mathrm{~km} / \mathrm{s}$, and it increases linearly as the depth increases. The maximum velocity is $4.5 \mathrm{~km} / \mathrm{s}$ at $3 \mathrm{~km}$ depth.

We perform refraction-traveltime tomography for two cases of the Marmousi-2 model using short- and large-aperture data. In both cases, 213 shots are located at the surface with intervals of $80 \mathrm{~m}$. For the short-aperture case, we locate 150 receivers with intervals of $20 \mathrm{~m}$ on the left side of every source simulating a 3000-m towed-streamer array. For the largeaperture case, 851 receivers are spread over the entire surface with spacings of $20 \mathrm{~m}$. In Figures $6 \mathrm{c}$ and $6 \mathrm{~d}$, we display the inverted models for the two cases of the Marmousi-2 model. From Figures $6 \mathrm{c}$ and $6 \mathrm{~d}$, note that the larger offset we use, the deeper structure we obtain. For comparison of our results with another inverted example, we also display an inverted velocity model derived from a simultaneous iterative reconstruction technique (SIRT) (Dines and Lytle, 1979) in Figure 6e. In the SIRT, we use the 80-m-grid Marmousi-2 model. In Figures $6 \mathrm{~d}$ and $6 \mathrm{e}$, we can see that our refraction tomography method gives results similar to those of the SIRT for the shal-

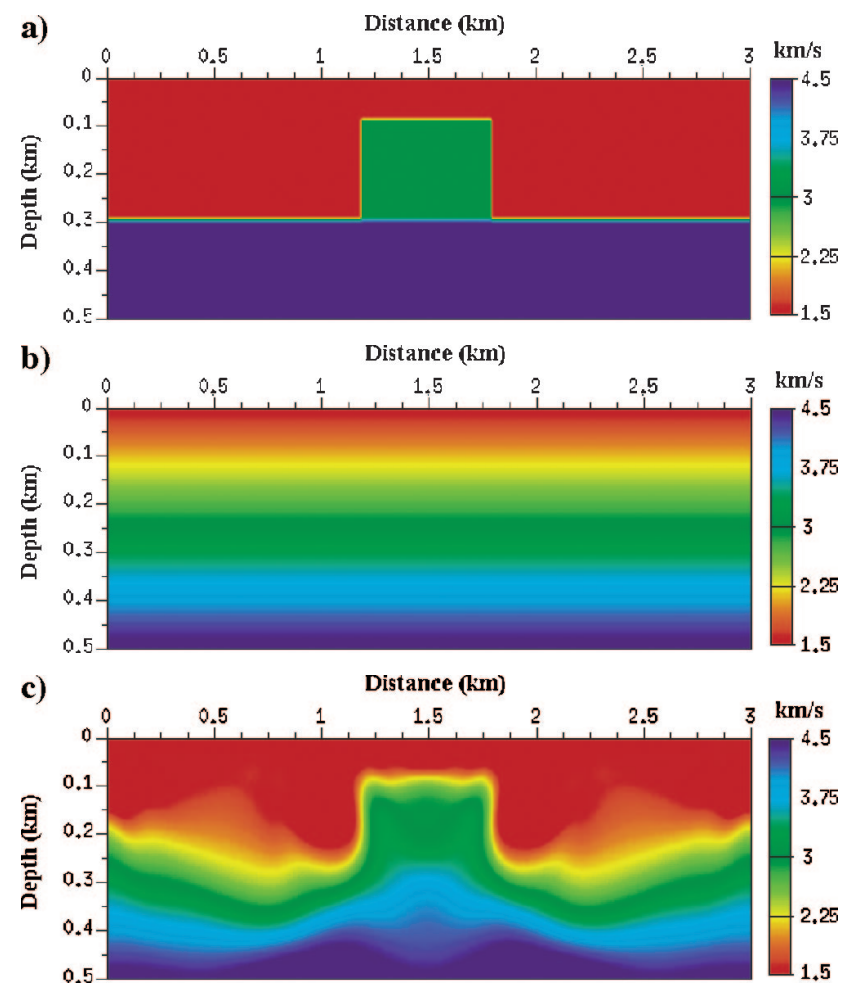

Figure 4. (a) An isolated block embedded in the two-layered model, (b) the initial model for the inversion, and (c) the last inverted model. low structures, but for the deep structures our method yields slightly better results.

Next, we examine whether the inverted velocity-depth model can be used as a smooth velocity model for the prestack depth migration. Figures $7 \mathrm{a}, 7 \mathrm{~b}, 7 \mathrm{c}$, and $7 \mathrm{~d}$ show the Kirchhoff-migrated images obtained by using the true, the initial, and the two inverted velocity models (for short- and largeaperture data) shown in Figures $6 \mathrm{a}, 6 \mathrm{~b}, 6 \mathrm{c}$, and $6 \mathrm{~d}$, respectively. For Kirchhoff prestack depth migration, we computed the most energetic traveltimes following the method of Shin et al. (2003b). From Figure 7, we note that the two inverted velocity models yield images superior to the initial velocity
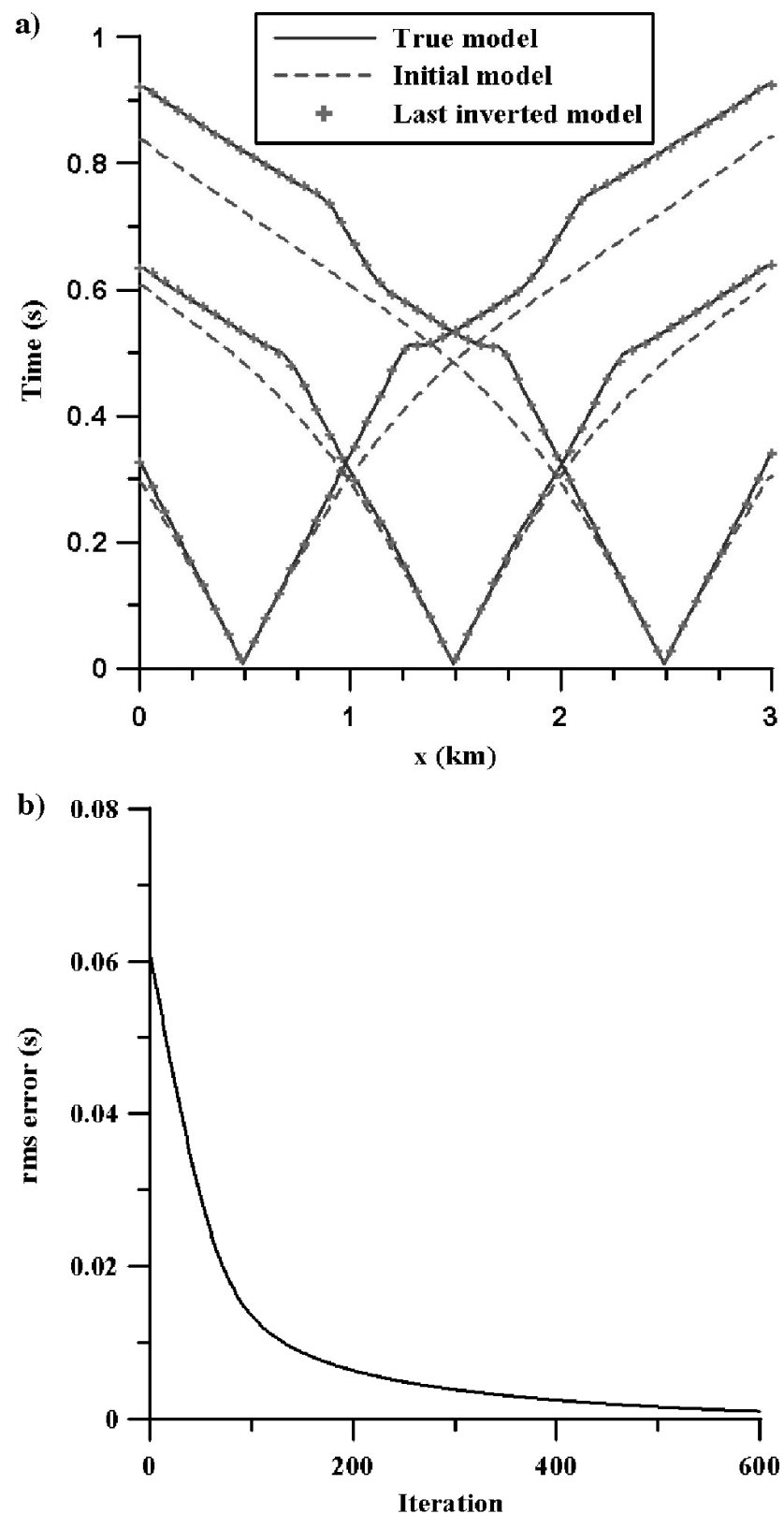

Figure 5. An isolated block embedded in the two-layered model: (a) the traveltime curves of the true model, the initial model, and the last inverted model, and (b) the history of rms error of our inversion results. 
model and that the large-aperture model gives the best image. In particular, the depth of the left side of the model is considerably improved, and the outline of the faults in the middle of the model is well defined. In addition, the continuity of the anticline in the deeper area is enhanced.

We also compare the Kirchhoff migration images obtained by our refraction tomography with those generated by the SIRT example. Figure 7e shows the Kirchhoff migration image obtained by using the SIRT. By comparing Figure 7d with Figure 7e, we note that the migrated image obtained by

a)

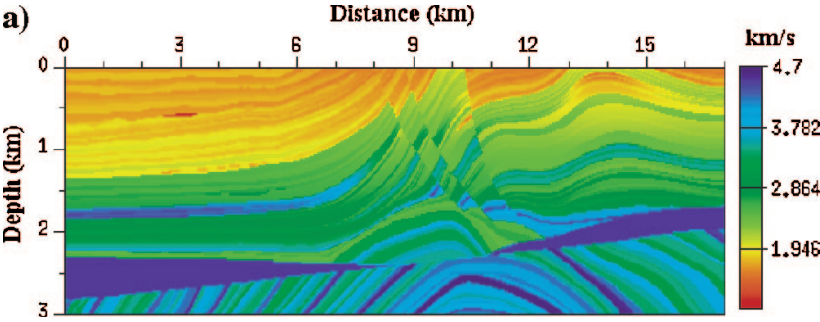

b)
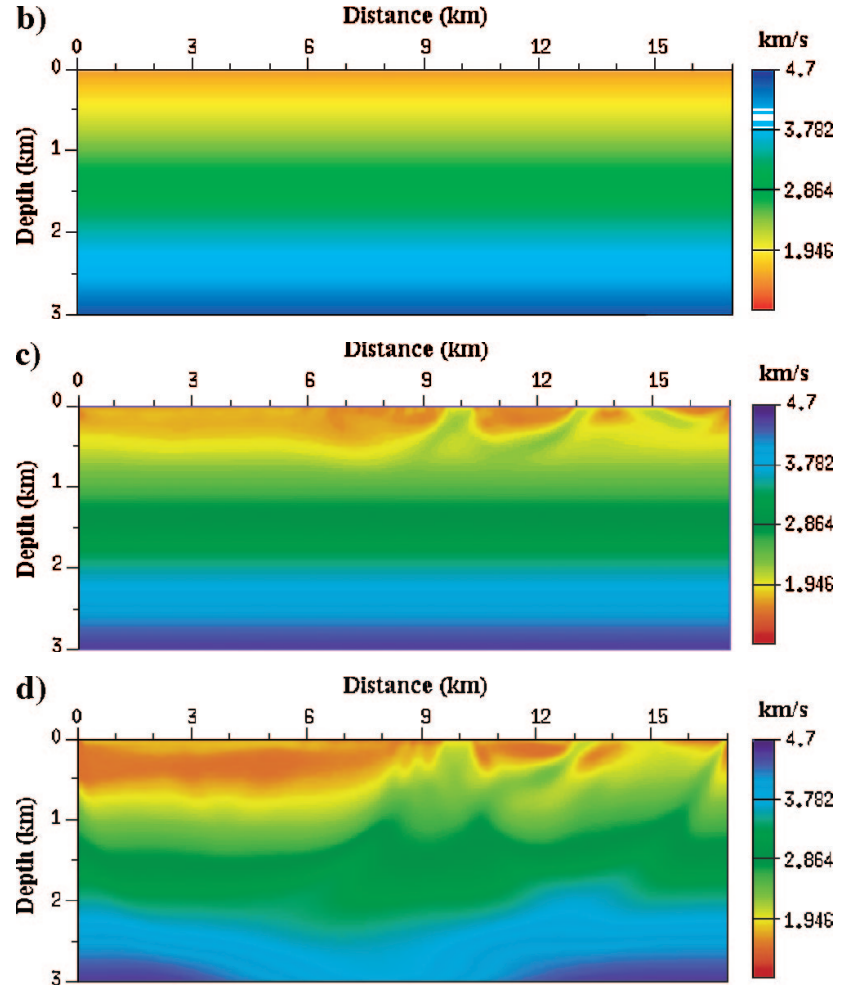

e)

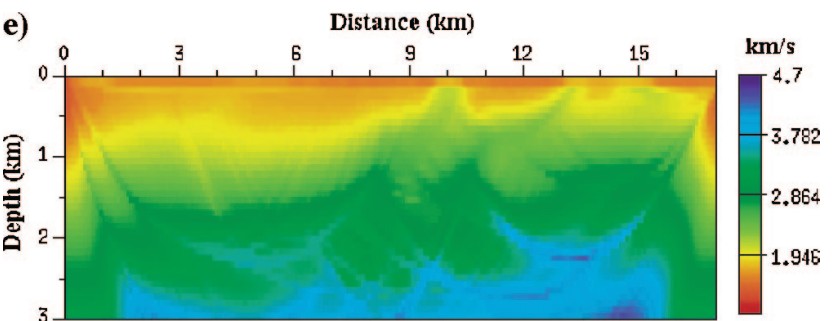

Figure 6. (a) The Marmousi-2 model, (b) the initial model used for the inversion, (c) the inverted model obtained using short-aperture data, (d) the inverted model obtained using large-aperture data, and (e) the inverted model obtained from the SIRT. our technique shows slightly better resolved structures for the deep part than those of the SIRT. From these results, we can safely assert that the inverted velocity model resulting from refraction-traveltime tomography can be satisfactorily used as
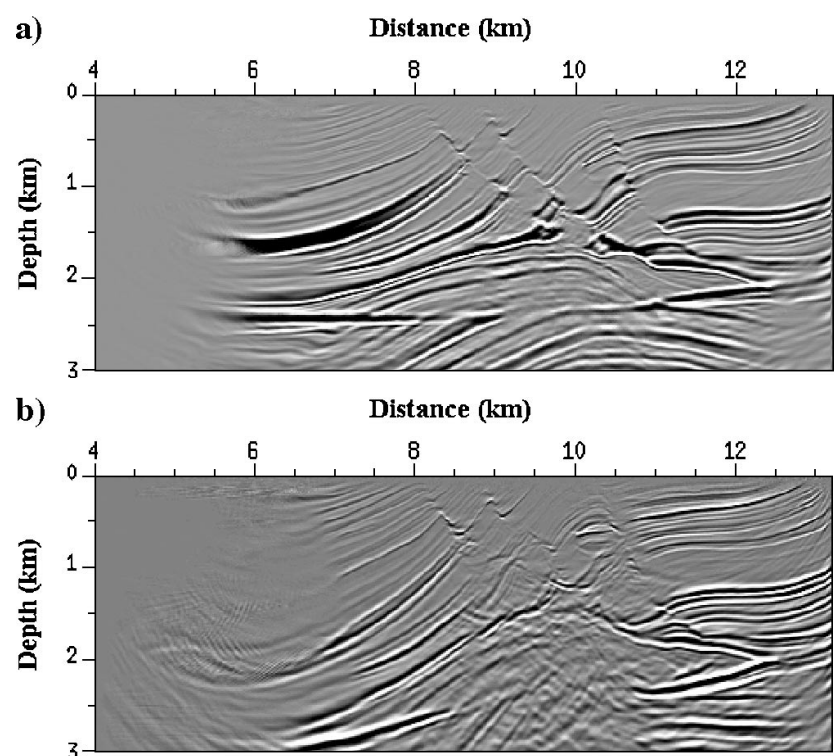

c)

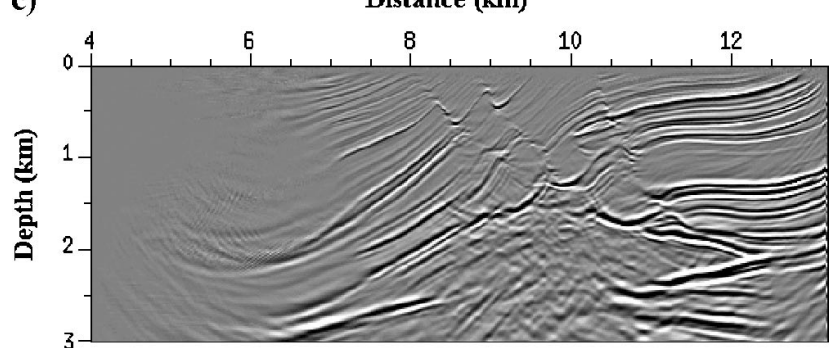

d)

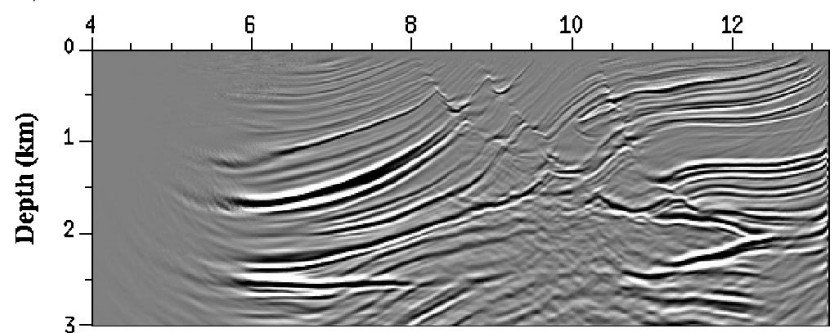

e)

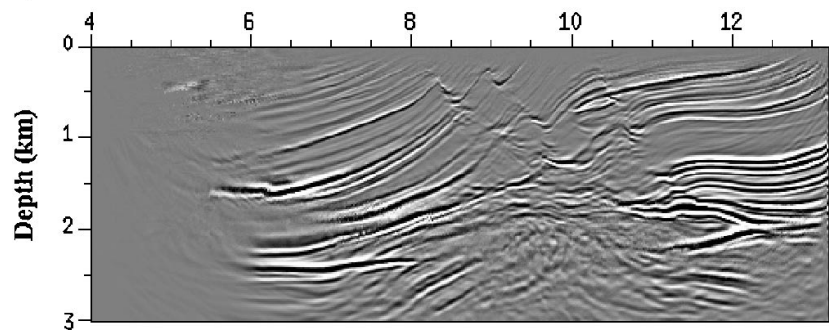

Figure 7. Kirchhoff prestack depth images generated for the Marmousi-2 model by using the most energetic traveltime calculated from (a) the ture model. (b) the initial model, (c) the inverted model obtained using the short-aperture data, (d) the inverted model obtained using the large-aperture data, and (e) the inverted model obtained from the SIRT. 
an initial velocity model for prestack depth migration, even though the accuracy of the velocity model decreases slightly at depth.

\section{CONCLUSIONS}

We developed a new traveltime-tomography technique using a monochromatic damped wavefield. An optimally damped wave equation propagates a single pulse instead of wave trains that are generated by a conventional two-way wave equation. By solving the damped-wave equation in the frequency domain and taking the logarithm of a damped wavefield at a single frequency, we extract the amplitude and the phase (or traveltime) of the first arrival. Because the damped-wave equation is symmetric, we apply the reciprocity theorem to reduce the computational cost required for computing Fréchet derivatives. We solve our inverse problem by using the steepest descent method and by regularizing the problem with the diagonal elements of the approximate Hessian, making it possible to use a scaled step length.

Our numerical tests of the Marmousi-2 model show that although refraction-traveltime tomography using large-aperture data does not give complete information on the subsurface, the velocity model obtained by refraction-traveltime tomography can be successfully used as a smooth velocity model for prestack Kirchhoff migration. Our refractiontraveltime tomography algorithm can also be used to delineate velocity structure for global tectonics and to estimate velocities in the shallow subsurface for engineering or environmental geophysics.

In the future, we expect that our algorithm for computing Fréchet derivatives of amplitude, as well as for traveltime, will allow us to include the amplitude term in refraction tomography and extend our algorithm to transmission tomography without any difficulty.

\section{ACKNOWLEDGMENTS}

This work was financially supported by the National Laboratory Project of the Ministry of Science and Technology and the Brain Korea 21 project of the Ministry of Education. We are also grateful to Prof. K. J. Marfurt of the University of Houston and Dr. M. Schoenberger for editing our manuscript.

\section{REFERENCES}

Cai, W., and F. Qin, 1994, Three-dimensional refraction imaging: 64th Annual International Meeting, SEG, Expanded Abstracts, 629-632.

Dines, K. A., and R. J. Lytle, 1979, Computerized geophysical tomography: Proceedings of the IEEE, 67, 1065-1073.
Docherty, P., 1992, Solving for the thickness and velocity of the weathering layer using 2-D refraction tomography: Geophysics, 57, 13071318 .

Hampson, D., and B. Russell, 1984, First break interpretation using generalized linear inversion: 54th Annual International Meeting, SEG, Expanded Abstracts, 532-534.

Lines, L. R., and S. Treitel, 1984, Tutorial: A review of least-square inversion and its application to geophysical problems: Geophysical Prospecting, 32, 159-186.

Luo, Y., and G. T. Schuster, 1990, Wave equation traveltime inversion: 60th Annual International Meeting, SEG, Expanded Abstracts, $1207-1210$.

- 1991, Wave equation traveltime inversion: Geophysics, 56 645-653.

Marfurt, K. J., 1984, Accuracy of finite-difference and finite-element modeling of the scalar and elastic wave equations: Geophysics, 49 533-549.

Martin, G. S., K. J. Marfurt, and S. Larsen , 2002, Marmousi-2: An updated model for the investigation of AVO in structurally complex areas: 72nd Annual International Meeting, SEG, Expanded Abstracts, 1979-1982.

Pratt, R. G., C. Shin, and G. J. Hicks, 1998, Gauss-Newton and full Newton methods in frequency domain seismic waveform inversions: Geophysical Journal International, 133, 341-362.

Qin, F., W. Cai, and G. T. Schuster, 1993, Inversion and imaging of refraction data: 63rd Annual International Meeting, SEG, Expanded Abstracts, 613-615.

Schneider, W. A., and S. Y. Kuo, 1985, Refraction modeling for static corrections: 55th Annual International Meeting, SEG, 295-299.

Schuster, G. T., and A. Quintus-Bosz, 1993, Wavepath eikonal traveltime inversion: Theory: Geophysics, 58, 1314-1323.

Shin, C., 1988. Nonlinear elastic wave inversion by blocky parameterization: Ph.D. thesis, University of Tulsa.

Shin, C., and S. Chung, 1999, Understanding CMP stacking hyperbola in terms of partial derivative wavefield : Geophysics, 64, 1774-1782.

Shin, C., J. Ha, and S. Jeong, 1999, Refraction tomography by blocky parameterization: Journal of Seismic Exploration, 8, 143-156.

Shin, C., S. Jang, and D. Min, 2001a, Improved amplitude preservation for prestack depth migration by inverse scattering theory: Geophysical Prospecting, 49, 592-606.

Shin, C., K. Yoon, K. J. Marfurt, K. Park, D. Yang, H. Lim, S. Chung, and S. Shin, 2001b, Efficient calculation of a partial-derivative wavefield using reciprocity for seismic imaging and inversion: Geophysics, 66, 1856-1863.

Shin, C., S. Ko, W. Kim, D. Min, D. Yang, K. J. Marfurt, S. Shin, and K. Yoon, 2003a, Traveltime calculations from frequency domain downward continuation algorithms: Geophysics, 68, 1380-1388.

Shin, C., S. Ko, K. J. Marfurt, and D. Yang, 2003b, Wave equation calculation of most energetic travel times and amplitudes for Kirchhoff prestack migration: Geophysics, 68, 2040-2042.

Shtivelman, V., 1996, Kinematic inversion of first arrivals of refracted waves-A combined approach: Geophysics, 61, 509-519.

Stefani, J. P., 1995, Turning-ray tomography: Geophysics, 60, 19171929.

Vasco, D. W., J. E. Peterson Jr., and E. L. Majer, 1995, Beyond ray tomography: Wavepaths and Fresnel volumes: Geophysics, 60, 17901804 .

White, D. J., 1989, Two-dimensional seismic refraction tomography: Geophysical Journal, 97, 223-245.

Woodward, M. J., 1992, Wave-equation tomography: Geophysics, 57 $15-26$.

Woodward, M. J., and F. Rocca, 1988, Wave-equation tomography: 58th Annual International Meeting, SEG, Expanded Abstracts, 1232-1235.

Zhang, J., and M. N. Toksöz, 1998, Nonlinear refraction traveltime tomography: Geophysics, $\mathbf{6 3}, 1726-1737$.

Zhu, X., and G. A. McMechan, 1989, Estimation of a two-dimensional seismic compressional-wave velocity distribution by iterative tomographic imaging: International Journal of Imaging Systems Technology, 1, 13-17. 
